\title{
Participation of Amygdalo-Hypothalamic Connections in the Regulation of The Hippocampal Theta Rhythm
}

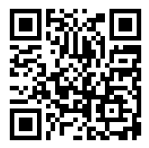

\author{
Rafiga Mazahir Baghirova* \\ Department of Medical and Biological Sciences, Azerbaijan State Academy of Physical Culture and Sports, Azerbaijan Republic
}

Received: July 21, 2018; Published: August 10, 2018

*Corresponding author: Rafiga Mazahir Baghirova, Department of "Medical and Biological Sciences", Azerbaijan State Academy of Physical Culture and Sports, Fatali Khan Khoyski Street, 98, Baku 1072, Azerbaijan Republic

\begin{abstract}
In chronic experiments on rabbits, we demonstrated that electrolytic destruction of the dorsal amigdalofugal way - stria terminalis results in intense depression of the integral electrical activity of the hippocampus. Morphological changes in neurons and satellite glial cells, such as lysis of the Nissl substance, hyperchromatosis, and wrinkling of the cells after destruction of the stria terminalis, were proof of the correctness of our conclusion. It is postulated that one of the main factors underlying the generalized effects observed in our experiments is probably the disturbance of functional characteristics of the hypothalamo- hypophyseal neurosecretory system induced by destruction of the amygdalofugal connections; this disturbance results in significant pathological shifts of metabolism in the hippocampus.
\end{abstract}

Keywords: Dorsal and Ventral Amigdalofugal Ways; Electrohippocampogram; Morphological Changes

\section{Introduction}

The study of the hippocampal theta rhythm is one of the urgent problems of modern neurophysiology. The medial septum nucleus, standing at the entrance to the hippocampus, demonstrates the importance of this education [1,2]. In addition to the data indicative of the pacemaker role of the septum, there are works which demonstrated the a definite role of stem-diencephalic structures in mechanisms of formation of hippocampal theta rhythm: of great importance given to the reticular formation [3], the hypothalamus [4], the thalamus [5], locus cereleus [6], nucleus raphe [7], etc. In order to study the role of amygdalo- hypothalamic connections in the processes of formation of the hippocampal theta rhythm in this study was conducted electrical stimulation and destruction of the dorsal (DAP) and ventral amygdalofugal pathways (VAP). After electrophysiological studies was conducted a morphological analysis in neurons and glial cells in various areas of the hippocampus and the medial nucleus of the septum.

\section{Methods}

Experiments were carried out on 20 mature rabbits weighing 2.5-3.0 kg. Electrostimulation and electrical destruction of the dorsal - DAP (precompassural region: A-1, L3.2, H11.5) and ventral - VAP (P5; L6H15.8) amygdalofugal pathways were made by bipolar electrodes. Both recording of the electrical activity from the hippocampus and septum and collection of the samplings for morphological studies were performed 18-27 days after such a destruction. The EHipG was recorded from the dorsal (the CA1 field: P 0 3.0, L 2.0, 1 8.0, and the CA3 field: P 0 2.0, L 6.0, 17.0 ) and ventral hippocampus (P4; L7; H17), from the dentate gyrus (P4; L5; N7,5) and from the medial nucleus of the septum (A -3.0; L 0.5; $\mathrm{H}$ 10.5 ) on a Medikor EEG-16E encephalograph with the use of needle electrodes insulated except the tip. For histological studies, brains of the animals were fixed in Carnoy solution and dehydrated using a series of alcohols of increasing concentrations; the blocks including the structures under study were embedded in paraffin. Frontal $10-\mu \mathrm{m}$-thick slices were prepared with a microtome, stained with $0.1 \%$ cresyl violet solution, treated with alcohol, cleared in xylene, embedded in balsam, and examined under a light microscope. The objects under study were neurons and glial cells of the hippocampus and septum

\section{Results and Discussion}

The results of these experiments showed that the baseline hippocampal and septal EEG demonstrated irregular activity dominated by oscillations in the range 4-6 Hz (Fig. 1 - I; 2 - I). In conditions electrostimulation both DAP and VAP at low parameters stimulating current (50-80 $\mu \mathrm{A}$, frequency $10 \mathrm{imp} / \mathrm{s}$, with a stimulus duration of $0.5 \mathrm{msec}$ ) is registered a clearly expressed synchronized activity, with a domination in the spectrogram of frequencies in the range of 6-7.5 $\mathrm{Hz}$ a decrease in high-frequency beta oscillations 
is observed and increase in amplitude of the investigated waves (Figures 1II \& 2II). The described changes in the EEG of the hippocampus were observed from the moment beginning electrical stimulation and persisted for 5-10 seconds after it was turned off. Further increase parameters of stimulation (100-200 $\mu \mathrm{A}$, frequency $20 \mathrm{~Hz}$ with a stimulus duration of $0.5 \mathrm{msec}$ ) for 10-15 seconds from the first minute's influence in the electrical activity of all registered structures simultaneously synchronized activity for 2-5 seconds of stimulation becomes epileptiforig. (Figures 1III \& 2III). Destruction of DAP (Figure 1IV) ways unlike VAP (Figure 2IV) leads to a complete and irreversible blockade of hippocampal theta rhythm (the longest observation time was six months).

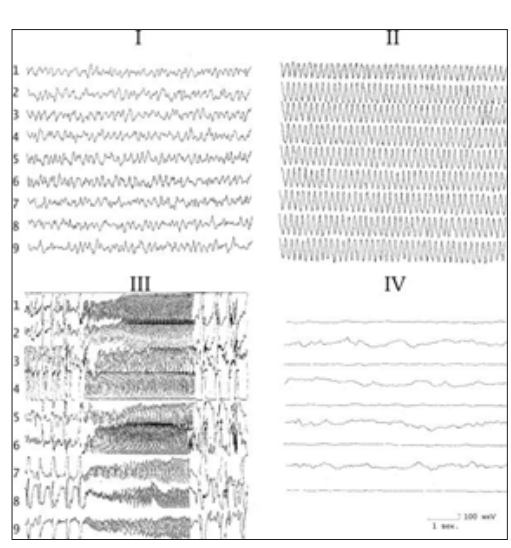

Figure 1: Changes in hippocampal electrical activity in rabbits under conditions of electrical stimulation and destruction of the DAP. I-background; II- after electrical stimulation at low parameters stimulating current; IIIafter electrical stimulation after increase parameters of stimulating current; IV-after destruction. 1,2)Field CA1; 34 ) field CA3 of the ipsi- and contralateral hemispheres; $5,6)$ ventral hippocampus of the ipsi- and contralateral hemispheres; 7,8 ) dentate gyrus of theipsi-and contralateral hemispheres; 9) medial nucleus of the septum.

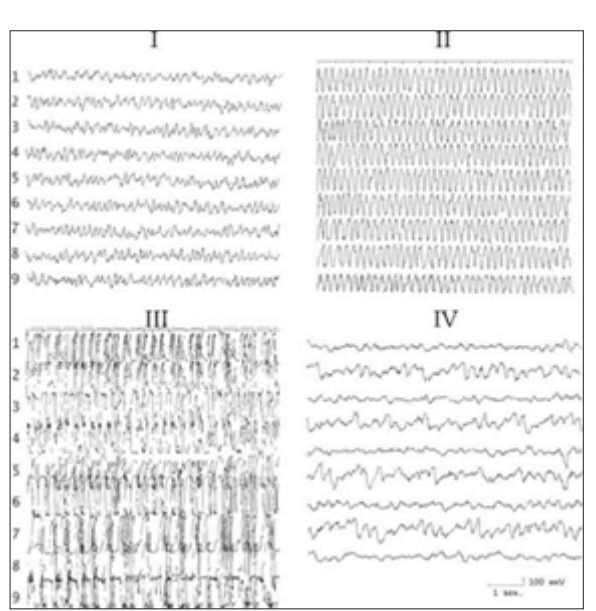

Figure 2: Changes in hippocampal electrical activity in rabbits under conditions of electrical stimulation and destruction of the VAP. The remaining designations the same as on page 1.

In the event of damage to the VAP, in contrast to the destruction of the DAP, complete recovery of EEG activity of the brain structures studied up to background level for 20- 25 days. Examination of the slices of the dorsal, ventral hippocampus, dentate gyrus and medial septum of experimental animals after coagulation of DAP demonstrated that profound morphological changes were detected in both neurons and glial cells of these regions (Figures 3-5). In particular, sections of the neuronal somata appear clear, i.e., lysis of the chromophilic matter (Nissl substance) was observed. A clearly pronounced edema of the apical dendrites was observed. In some neurons, in the volume of the latter were typical findings. In this case, basket-like cells within the CA1 and CA3 fields, as compared with the pyramidal neurons, underwent less pronounced morphological modifications, whereas the intensity of staining of the tigroid matter was preserved to a significant extent. In the medial septum, clearly pronounced hydropic changes were also observed; they were especially expressed in the multipolar neurons.

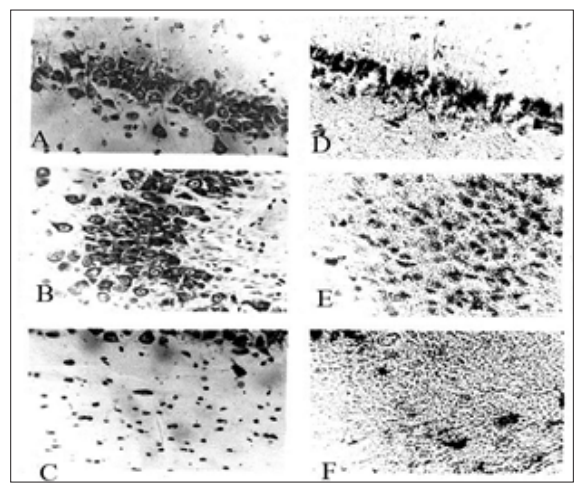

Figure 3: State of the Nissl substance in neurons and glia of the hippocampus and dentate gyrus before and after destruction of the DAP. A,B,C - CA1 and CA 3 fields and dentate gyrus before; D,E,F - after destruction.

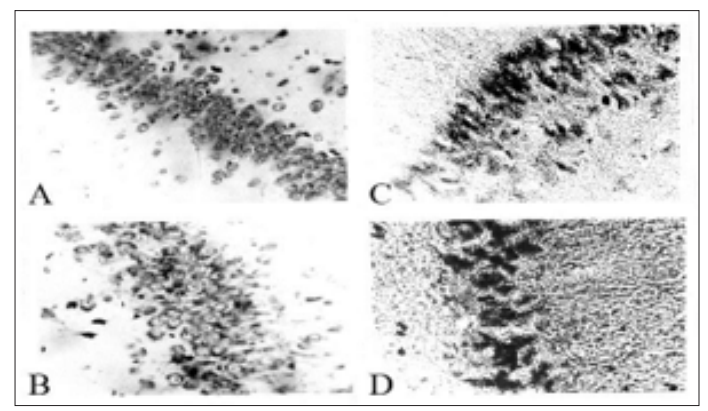

Figure 4: State of the Nissl substance in neurons and glia of the ventral hippocampus before and after destruction of the DAP. A,B - CA1 and CA 3 fields before; C, D - after destruction of the dorsal amygdalofugal pathway.

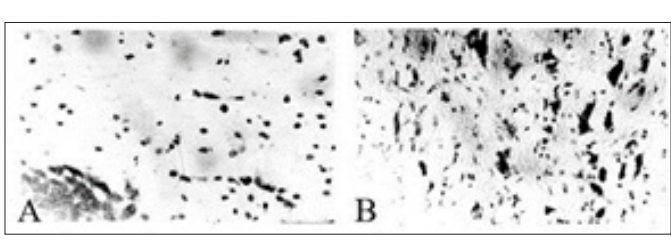

Figure 5: State of the Nissl substance in neurons and glia of the medial septal nucleus before and after destruction of the DAP. The medial nucleus of the septum before (A) and after destruction (B). 
The cross sections of many cells looked like transparent contours, where the tigroid almost disappeared. Cellular borders of the individual neurons were smeared, and the nuclei were distorted and compressed. In the cytoplasm, numerous vacuoles and manifestations of edema of the somata, nuclei, and cell processes were observed. In glial cells, clearness and swelling of the somata was observed. In thecytoplasm of some glial cells, light vacuoles were seen. In the karyoplasm of glial cells, light zones were observed. We also observed clearness of the cytoplasm around the nuclei due to redistribution of the tigroid substance and its displacement toward the periphery. The possibility for modulation of the electrical activity of the hippocampus, and especially of the EHipG theta rhythm, because of changes in the functional state of extrahippocampal structures of the brain (including the hypothalamus and amygdala) was demonstrated earlier [2,3,5]. A basic aspect of the data we obtained in our experiments is that we demonstrated deep and longlasting depressive modulation of the mass electrical activity of the hippocampus under conditions of coagulation of the stria terminalis. Our data allow us to believe that the above depression is related to changes in the functional state of hippocampal neurons themselves, but not to stria terminalis destruction determined modification of the pattern and intensity of afferent impulsation coming from extrahippocampal structures.

Histological observations, according to which deep changes occur in the structure of hippocampal neurons and satellite glia after coagulation of the stria terminalis, also confirm the conclusion of decreased excitability of the neurons in the septohippocampal system. Recovery of electrical activity in the form of regular oscillations only in response to factors inducing stable neuron membrane depolarization may be evidence primarily of profound metabolic derangements occurring simultaneously in all hippocampal fields and indicating the diffuse, neurohumoral nature of changes resulting from lesioning of the amygdalofugal connections. It can be suggested that the long-term depression of the hippocampal EEG observed here results from disturbance to the normal functioning of the hypothalamo-hypophyseal neurosecretory system due to lesioning of the stria terminalis, which ultimately leads to long-lasting changes in the excitability of hippocampal neurons. However, whatever the mechanism of EEG depression in this experimental situation, it is clear that the integrity of the dorsal amygdalofugal pathway is one of the key factors in regulating neuron excitability in the septohippocampal system.

\section{References}

1. Kichigina VF, Kudina TA (2001) Sensory reaction of hippocampal neurons of the rabbit under functional you turn off the structures that control theta rhythm. J Higher Nervous Activity 51(2): 228-235.

2. Kitchigina VF, Kutireva ES, Sudnitsin VV (2005) Sensory responses of medial septal neurons during theta activity modulation by agonist alpha2-adrenoreceptors clonidine. J Neurosci Behav Physiology 35: 107-117.

3. Kichigina VF, Kudina TA, Zenchenko KI, Vinogradova O (1998) Background activity of hippocampal neurons of the rabbit after switching off the structures controlling the theta rhythm. J Vyssh Nerv Deyat 48(3): 505-509.

4. Smythe JW, Christie BR, Colom LV (1991) Hippocompal theta field activity and theta-on/theta-off all discharges are controlled by an ascending hypothalamo-septal pathway. Neuroscience 11(7): 2241-2248.

5. Kichigina VF, Kutyreva EV (2002) Modulation of theta activity in septohippocampal system alpha agonist clonidine 2 adrenoceptor. Journal higher nervous activity 52(2): 195-204.

6. Berridge CW, Espana RA (2000) Synergistic sedative effects of nonadrenergic $\alpha 1-$ and $\beta$-receptor blockade in forebrain electroencephalographic and behavior indices. J Neuroscience 99(3): 495-505.

7. Kudin KN, Zenchenko et al. (1998) J Higher Nervous Activity 48(3): 505515.
ISSN: 2574-1241

DOI: 10.26717/BJSTR.2018.07.001562

Rafiga Mazahir Baghirova. Biomed J Sci \& Tech Res

This work is licensed under Creative Commons Attribution 4.0 License

Submission Link: https://biomedres.us/submit-manuscript.php

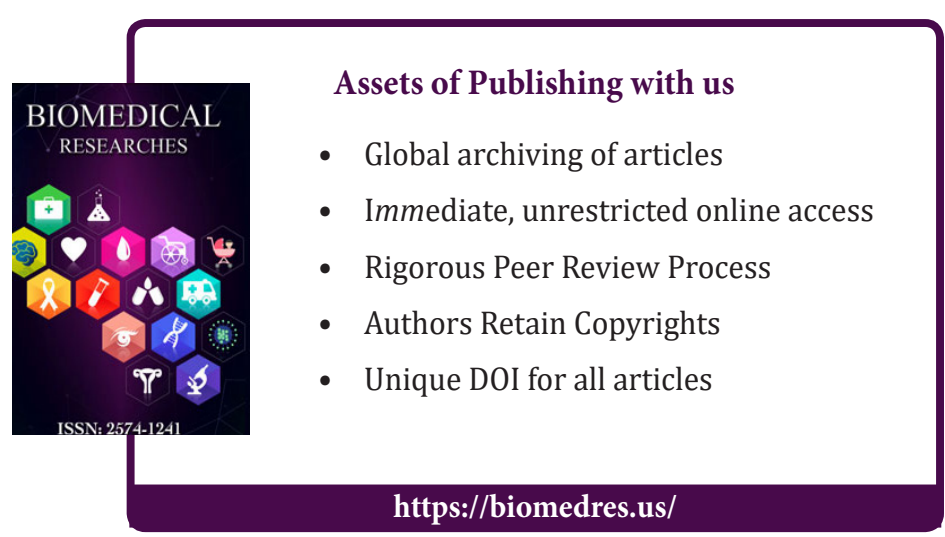

